\title{
The trunk and pelvis rotation range of motion and coupled motions in the sagittal plane in adolescent girls with idiopathic scoliosis Agnieszka Stępien* ${ }^{*}$ and Andrzej Seyfried
}

\author{
Address: Department of Rehabilitation, University of Physical Education in Warsaw, Poland
}

* Corresponding author

from $5^{\text {th }}$ International Conference on Conservative Management of Spinal Deformities

Athens, Greece. 3-5 April 2008

Published: 15 January 2009

Scoliosis 2009, 4(Supp| I):O9 doi:I0.I|86/I748-7|6I-4-SI-O9

This abstract is available from: http://www.scoliosisjournal.com/content/4/SI/O9

(C) 2009 Stêpieñ and Seyfried; licensee BioMed Central Ltd.

\section{Introduction}

Contemporary medicine tends to analyze scoliosis in 3-D. A spine motions in one plane are coupled with motions in others planes. The purpose of this study was to analyze the trunk and pelvis rotation ROM in horizontal plane and coupled motions in sagittal plane in scoliotic girls.

\section{Hypothesis}

Scoliosis causes changes of rotation ROM according to healthy subjects. Trunk and pelvis rotation are coupled with motions in sagittal plane. Rotation ROM and coupled motions in sagittal plane depend on a type of scoliosis.

\section{Materials and methods}

Fifty nine AIS girls and 30 healthy girls at the age 10-18 participated in the study. A right thoracic curve and/or a left lumbar curve at the spine radiography were criterions for studies. Girls were assigned to four groups according to type of scoliosis. A computer-based axial rotation test assessed the trunk and the pelvis rotation ROM and coupled motions in the standing, upright position. The thoracic cage or the pelvis were fixed during tested motions. ANOVA test and T-test were used in statistic analyze.

\section{Results}

There are differences of rotation ROM in scoliotic subjects comparing to the control group. A decrease in ROM of the trunk and pelvis rotation in double curve scoliosis girls was observed. Increasing ROM of pelvis rotation in left lumbar curves subjects was found. The right thoracic curve is correlated with decreasing ROM of left trunk rotation and the left lumbar curve is associated with increasing ROM of left pelvic rotation. Right rotation of the trunk is correlated with extension in thoracic curve subjects and right rotation of the pelvis is coupled with increasing anterior pelvic tilt in all formed groups. Increasing trunk extension was observed in scoliotic subjects comparing to the control group.

\section{Conclusion}

Documented relationships of the trunk and pelvis motions in horizontal and sagittal planes contribute to development of knowledge of AIS biomechanical backgrounds. This knowledge is necessary to plan the most effective way of treatment in AIS patients.

\section{References}

I. Kotwicki T, Walczak A, Szulc A: Trunk rotation and hip joint range of rotation in adolescent girls with idiopathic scoliosis: does the "dinner plate" turn asymmetrically? Scoliosis 3:I. 2008 Jan 19; 